\title{
Review of the British Thoracic Society Winter Meeting 2012, 5 to 7 December, London, UK
}

\author{
Hannah Kelly Bayes, ${ }^{1}$ Alistair Colin Church, ${ }^{2}$ Andrew J Fisher, ${ }^{3}$ on behalf of the BTS \\ Science and Research Committee
}

${ }^{1}$ Department of Inflammation and Immunology, Institute of Infection, University of Glasgow, Glasgow, UK ${ }^{2}$ Department of Respiratory, Scottish Pulmonary Vascular Unit, Glasgow, UK

${ }^{3}$ Institute of Cellular Medicine, Newcastle University, Newcastle, UK

\section{Correspondence to} Professor Andrew J Fisher, Institute of Cellular Medicine, Newcastle University Medical School, Framlington Place, Newcastle Upon Tyne NE2 4HH, UK; a.j.fisher@ncl.ac.uk

HKB and ACC contributed equally

Received 19 December 2012 Revised 19 December 2012 Accepted 20 December 2012 Published Online First 12 January 2013

To cite: Bayes HK, Church AC, Fisher AJ, et al. Thorax 2013;68:277-282.

\section{ABSTRACT}

This review highlights new developments in scientific and clinical research presented at the British Thoracic Society Winter Scientific Meeting held from 5 to 7 December 2012. Although a wide spectrum of respiratory research was presented at the meeting the content of the review focuses specifically on the key themes of pleural disease, interstitial lung disease and future therapies in respiratory medicine. Advances in clinical and translational respiratory research presented in the major symposia and spoken sessions related to these areas are summarised. Additional sessions covering lifestyle dilemmas in the context of respiratory disease and the early career investigator awards are also highlighted.

This review aims to encapsulate the exciting and important new developments in respiratory medicine presented at the annual British Thoracic Society (BTS) Winter Scientific Meeting. As a significant breadth of scientific and clinical research was presented at the 23 major symposia and in 430 submitted abstracts, the review focuses on key themes: future therapies in respiratory medicine, pleural disease, and interstitial lung disease (ILD). Additional well received sessions covering lifestyle dilemmas in the context of respiratory disease and the early career investigator awards are also highlighted.

\section{EVOLVING THERAPIES FOR RESPIRATORY DISEASE}

This year's BTS conference included several excellent sessions highlighting novel treatments and means of drug delivery in respiratory disease. A two-part symposium run in association with the British Association for Lung Research (BALR) covered emerging strategies of therapeutic delivery to the lungs. Inhaled therapies date back to the ancient Egyptians, however Dr Omar Usmani (London) discussed how effective drug delivery to the lungs remains problematic, with current devices achieving only $10-20 \%$ lung deposition. ${ }^{1}$ In addition confusion over inhaler devices remained prevalent in both healthcare professionals and patients, which was described separately by Mak et $a l^{2}$ in a survey of over 1200 healthcare professionals. Dr Usmani highlighted that the input of design engineers and formulation chemists had now resulted in introduction of innovative inhaler devices, an example being intelligent nebulisers which adapt to breathing patterns to achieve 50$70 \%$ drug deposition, and manipulation of particle size to allow effective treatment of small and large airways. $^{3}{ }^{4}$ Work is also ongoing to use the lungs, with their rich vascular bed, to deliver a range of therapies including inhaled chemotherapy, vaccines, biologicals and analgesics.

Nanomedicine uses the enhanced magnetic and electrical properties and increased surface area: volume ratio of particles less than $100 \mathrm{~nm}$ in size. Dr Andrew Thorley (London) provided examples of inhaled nanomedicine currently in development. Inhaled anti-tuberculosis nanomedicines allow delivery to the site of disease, potential for phagocytosis by macrophages and formulation to allow delivery as a single combination therapy, with promising preclinical in vivo work. Cancer therapeutics may be particularly amenable to nanomedicine due to increased permeability of tumours to nanosized particles and enhanced retention of agents due to poor vascular and lymphatic drainage. For example, tumour necrosis factor $\alpha(\mathrm{TNF} \alpha)$ coated gold nanoparticles reduce tumour size in murine models and have now entered phase I clinical trials. Finally, with manmade and natural nanoparticles eliciting toxic effects, ${ }^{5}$ Dr Thorley urged judicious use of nanomedicine to balance beneficial and potential adverse effects.

Gene therapy for cystic fibrosis lung disease has explored both virus and liposome-mediated lung delivery. Professor Eric Alton (London) presented the scientific and clinical research programme of the UK CF Gene Consortium (http://www.cfgenetherapy.org. uk). This has lead to a trial of liposome-delivered gene therapy in patients over 12 years of age who receive multidose treatment over a 12 -month period, the results of which are expected in spring 2014. Professor Alton also described promising results from the next stage in gene therapy via the use of a modified lentivirus, which achieves significantly better and sustained delivery compared with liposome-mediated treatment.

Moving from the current therapies available, the second part of this symposium detailed advances in use of cells as delivery systems to areas of disease. Professor Claire Lewis (Sheffield, UK) detailed the identification of tumour active macrophages (TAMS), which home to areas of hypoxia and their manipulation to target lung metastases of prostate cancer. The macrophages were cotransfected with an adenoviral E1A-dependent oncolytic virus and a hypoxia-inducible factor (HIF)-responsive plasmid encoding E1A. When the transfected macrophages were introduced into a murine model with a prostate tumour attached, the cells homed to the hypoxic areas within the tumour. This activated the HIF-responsive genes and led to upregulation of EIA and replication of the virus. However, for the 
virus to be able to further replicate it requires the cell to contain a prostate-specific factor, thus limiting further viral replication to prostate tissue. Convincing data were presented showing tumour regression and prevention of metastases after introduction of these TAMS into the mice. This seems a very exciting and novel method to allow the introduction of tumouricidal cells to target a cancerous cell population.

Next Dr Sam Janes (London) discussed mesenchymal stem cells (MSCs) as a potential vehicle for delivery of TNF-related apoptosis inducing ligand (TRAIL) to a murine model of lung cancer. Again these cells home to areas of inflammation and damage and within $24 \mathrm{~h}$ of administration mesenchymal cells were identified within the tumour. Although the size of the tumour was not reduced there was a definite reduction in the number of pulmonary satellite metastases observed within the animals. This approach was also presented separately by Sage $e t a l^{6}$ in the context of mesothelioma. These presentations suggest an exciting new opportunity for treating lung cancer.

Finally, Professor John Simpson (Newcastle, UK) described the alternative approach of targeting cell removal. He exemplified this via experimental data showing that removal of blood monocytes can result in a reduction of the development of acute lung injury from inhaled lipopolysaccharide in murine models. The proposed mechanism being reduced recruitment of neutrophils to sites of inflammation due to loss of monocytederived chemoattractants. However, in subsequent human experiments, removal of monocytes by plasma leucopheresis did not alter neutrophil numbers isolated at bronchoscopy from the lungs of subjects exposed to lipopolysaccharide. Thus Professor Simpson reminded us of the importance of pairing murine models with human studies.

\section{LUNG CANCER THERAPEUTICS}

A separate symposium was dedicated to the evolving use of personalised medicine in lung cancer. Dr Martin Forster (London) and Dr Sara Busacca (Leicester, UK) described the current situation regarding targeted therapy in non-small cell lung cancer (NSCLC). This includes the first-line use of epithelial growth factor receptor (EGFR) tyrosine kinase inhibitors in patients with NSCLC harbouring the mutation, targeting of progression due to a second mutation in EGFR mutation positive disease, and recent work targeting anaplastic lymphoma kinase (ALK) rearrangements via use of the selective ALK inhibitor crizotinib. ${ }^{78}$ Dr Forster concluded that with the ongoing development of targeted therapies for lung cancer there will be a need for services to examine the molecular background in patients, means to serially examine tumours during therapy and studies designed to test treatments in small, highly selected subpopulations of patients. Dr Busacca also highlighted the potential to use circulating cancer DNA from peripheral blood samples to examine mutation status and thus guide therapy. Finally, Dr Nirupa Murugaesu (London) discussed tumour heterogeneity as a potential bar to personalised lung cancer treatment. She eloquently described the complexity of mutations present in a single tumour. Although the dominant mutation may be treated via current targeted therapy, additional low-frequency mutations drive resistance and effect outcome (eg, presence of pretreatment T790M mutations predict shorter tyrosine kinase inhibitor response in NSCLC). Current work by her group aims to further educate us in the clonal evolution of NSCLC.

\section{SEVERE ASTHMA THERAPEUTICS}

Severe asthma poses a significant burden on healthcare resources, with the BTS Difficult Asthma Registry reporting at this meeting that over $50 \%$ of these costs are attributable to drug costs. ${ }^{10}$ New therapies are emerging, for example, antiinterleukin (IL)-13 and bronchial thermoplasty, but will require judicious use. Dr Andrew Menzies-Gow (London) highlighted the need for asthma biomarkers to help direct these novel therapies. This was exemplified by the recent use of the systemic biomarker periostin which predicts eosinophilic airways disease $^{11}$ and can be used to direct therapies targeting $\mathrm{T}$ helper 2 disease, including inhaled corticosteroids and the anti-IL13 monoclonal antibody, lebribizumab. ${ }^{12}$ Bronchial thermoplasty is now being performed in the clinical setting for severe asthma, as described separately by Bicknell et al. ${ }^{13}$ However, Dr Menzies-Gow stressed there is a pressing need to develop better biomarkers to predict response to such treatment. In a complimentary session, Professor Richard Knowles (Stevenage, UK) described the U-BIOPRED project, which is collecting biochemical, genetic and clinical data from infants with recurrent wheeze through to adults with asthma (http://www.ubiopred. european-lung-foundation.org). ${ }^{14}$ Such work should enable improved phenotyping of patients with asthma, development of biomarkers and direction of novel therapies.

\section{PLEURAL DISEASE}

There is increasing interest in the area of pleural disease and this was reflected in a number of excellent sessions dedicated to that topic. Although relatively uncommon (10\% of parapneumonic effusions are complicated or frankly infected), empyema is associated with poor outcome. The overall mortality from empyema is $20 \%$ at 1 year and death of surgery occurs in over $20 \%$ by 3 months; such adverse outcomes have remained unchanged over the last 20 years. Dr Naj Rahman (Oxford, UK) updated the BTS on the evidence supporting intrapleural fibrinolytics in the treatment of empyema and complicated parapneumonic effusions. He explained that the conclusions of the Multicenter Intrapleural Sepsis Trial (MIST1), ${ }^{15}$ demonstrating that streptokinase alone was ineffective in pleural infection, may have been effected by failure of the agent to alter pleural fluid viscosity $^{16} 17$ and biofilm formation. The recently reported Multicenter Intrapleural Sepsis Trial (MIST2) confirmed that fibrinolytics alone are ineffective, but that the combination of tissue plasminogen activator (tPA) and DNase results in effective drainage of the pleural space (with a $29.5 \%$ reduction in the area of hemithorax occupied by pleural fluid on chest $\mathrm{x}$-ray compared with a $17.2 \%$ reduction with placebo; $p=0.002) .{ }^{18}$ There were also suggestions of reduced surgical intervention, length of hospital stay and occurrence of sepsis syndrome in the tPA/DNase group. However, Dr Rahman suggested that the outcomes of hospital stay and mortality would now be more rigorously tested in a subsequent trial.

The incidence of pleural infection is increasing worldwide in the paediatric and adult populations, ${ }^{19} 20$ with several talks discussing the bacteriology and pathogenesis of these infections. Dr Nicholas Maskell (Bristol, UK) discussed the discordance between the bacteriology of pneumonia and that of pleural infection. He provided evidence that approximately $30 \%$ of empyema cases are not associated with parenchymal consolidation on CT and the bacteriology of pleural infection corresponds with that of the oral cavity. Dr Maskell thus proposed that two processes might lead to pleural infection, either as a complication of pneumonia or via translocation of oral bacteria into the airways and then invasion of the pleural space. Professor Gary Lee (Perth, Australia) also highlighted the challenges in understanding the pathogenesis of pleural infection. ${ }^{21}$ Using a murine model, his group have shown that following intranasal 
administration Streptococcus pneumoniae can breach the visceral pleural surface and upon entry the pleural cavity offers a protected compartment for bacterial growth with ineffective local defence mechanisms. ${ }^{22}$ In addition, Professor Lee discussed potential strategies to improve the poor microbiological yield in empyema (with $40 \%$ of causative organisms not currently being identified), including whether bacterial yield may be increased by use of sterile containers and blood culture bottles, ${ }^{23}$ pleural biopsy and identification via nucleic acid amplification. ${ }^{24}$

The UK-Enhanced Surveillance of Pneumococcal Empyema (UK-ESPE) study, which aims to monitor the changing epidemiology of paediatric empyema and to compare empyema management across the UK, was presented by Dr David Spencer (Newcastle, UK). This demonstrated pneumococcus as the most commonly identified cause of paediatric empyema, although the causative serotypes had evolved since the introduction of pneumococcal vaccines. Dr Spencer highlighted an increase in serotype $19 \mathrm{~A}$ disease in the $\mathrm{UK}^{25}$ and internationally, ${ }^{26}$ which is associated with increased virulence and poorer outcomes. In a spoken session, Corcoran et $a l^{27}$ also reported their review of the literature demonstrating that pleural infection was predominantly due to Steptococcus milleri and pneumoniae, but that there are substantial variations in bacteriology depending on where infection is acquired, locally (nosocomial vs community) and globally. Such results have important implications for antibiotic choice, with a need to identify the most prevalent organism in your local region.

The interest in pleural disease was further reflected in several excellent spoken and poster abstract sessions. Following on from the MIST- 2 trial, Pitman et $a l^{28}$ presented work by the Glasgow group on the use of tPA and DNAse in empyema management in clinical practise. This showed that in patients who had not responded to standard management with chest drain insertion and saline flushing, that the addition of tPA/DNAse led to increased drainage, improvement in the radiological appearances and a reduced requirement for surgical input. In addition, Hooper $e t$ al $^{29}$ showed that by using $250 \mathrm{ml}$ saline three times a day, direct pleural irrigation could improve radiological appearances as determined by CT and also reduced surgical referral. This was well tolerated and cost effective.

One of the chest physicians' worst nightmares is a difficult drain falling out of a patient, usually overnight! In a novel approach, Wilkinson examined why this may occur by using a model which allowed him to test an operator's ability to secure a $12 \mathrm{~F}$ chest drain to a plasterboard using sutures of the operator's choice. Then using a Newton metre the force required to remove it was assessed. He found that using larger sutures was the best, and at least in his model, a modified adhesive dressing could be used with similar efficacy. ${ }^{30}$ Finally, Buckley et $a l^{31}$ looked at trying to develop a scoring system for predicting pleural malignancy from a CT scan and the need for further investigation.

\section{INTERSTITIAL LUNG DISEASE}

Urgent new therapies are required for many of the ILDs. This year the BTS hosted a number of key symposia and sessions on this very topic, including an imaginatively titled session 'Scar wars'. This state of the art symposium began with Professor Richard Hubbard (Nottingham, UK) detailing the epidemiological evidence of the potential involvement of the clotting cascade in the development and progression of idiopathic pulmonary fibrosis (IPF). Previous studies have suggested that the presence of a prior episode of venous thromboembolism or being on anticoagulation can result in a three times greater risk of developing IPF. Indeed a separate spoken presentation by Navaratnam et $a l^{32}$ corroborated this finding. However, the ACE-IPF (AntiCoagulation Effectiveness in IPF) trial has shown that using warfarin in IPF management led to increased exacerbation rates and death, unrelated to drug side effects, in the treatment group. ${ }^{33}$ Thus the role of clotting in IPF is far from clear.

This was followed by an informative talk from Professor David Schwartz (Colorado, USA) on the genetics of IPF and the identification of the MUC5B gene as a risk factor. Having a genomic variant in the MUC5B promoter, leading to an altered transcriptional factor binding site, is associated with an increased risk of developing a spectrum of interstitial lung abnormalities on CT. This variant seems to be common in certain epidemiological studies but is associated with a milder form of the disease. Professor Derek Mann (Newcastle, UK) then spoke elegantly on the role that epigenetics might play in the pathogenesis of pulmonary fibrosis. Using his work in liver fibrosis as an exemplar, he produced clear evidence that methylation of key signature sequences in the 'methylome' of a hepatic stellate cell can lead to its differentiation into a myofibroblast with subsequent disordered collagen production and organ fibrosis. ${ }^{34}$ Inhibition of this methylation can prevent this differentiation, which may also be a strategy to investigate in pulmonary biology.

All cells operate in an organised structure called the extracellular matrix and Professor Jack Gauldie (Hamilton, Canada) gave a fascinating overview of the importance of this structure. Fibroblasts which have been added to matrix derived from patients with IPF are stimulated to differentiate into myofibroblasts-the cells sense the environment around them and respond to it. Furthermore mechanical signalling through the extracellular matrix can result in the proliferation of certain cells. Thus the matrix has to be viewed as a dynamic environment, which can result in cellular effects that can be pro-fibrotic.

In a spoken session, Maher et al presented an outline of the early clinical real-world experience with pirfenidone in the UK. ${ }^{35}$ They showed it to be reasonably well tolerated and in over $50 \%$ of patients who did develop an adverse effect a reduction in dosage allowed the drug to be continued.

There was an excellent update in a dedicated symposium covering uncommon ILDs. Professor Simon Johnson (Nottingham, UK) gave an in-depth discussion on lymphangioleiomyonmatosis (LAM). Of key interest was the fact that serum levels of vascular endothelial growth factor D are elevated in patients with LAM and that this may be incorporated into future diagnostic criteria. Furthermore the translational journey leading from the identification of mutations in the tuberous sclerosis complex genes (TSC-1 and TSC-2) as being responsible for this disease, through to the novel treatment with mammalian target of rapamycin inhibitors were presented. ${ }^{36}$ In addition, these patients can develop angiomyolipomas and Yeoh et $a l^{37}$ presented a study showing that they are common and require intervention more frequently when arising in the TSC-associated LAM.

Dr Morgan (London) delivered a very informative talk on the diagnosis and management of pulmonary alveolar proteinosis. This included detailing the underlying autoimmune process with anti-granulocyte macrophage colony-stimulating factor (GM-CSF) antibodies neutralising the alveolar expression of GMCSF, which leads to altered cellular signalling and release of increased levels of surfactant. The mainstay of treatment remains whole lung lavage, which involves the washout of the affected lungs using large amounts of saline, and has kept 
people alive who otherwise would have succumb to the disease. More recently the use of inhaled GM-CSF has resulted in substantial improvement in patients' quality of life with a reduction in the need for whole lung lavage. ${ }^{38}$

Professor Anthony Chu (London) manages patients with systemic Langerhans cell histiocytosis ( $\mathrm{LCH}$ ) and highlighted that this disease has a myriad of presentations. Diagnosis of pulmonary LCH rests with high-resolution CT and lung biopsy. However, a bronchoalveloar lavage with greater than $5 \%$ of CD1a positive cells can be very suggestive. Although smoking cessation is important, the evidence does not suggest that this alone is the only treatment. Indeed immunosuppression with steroids, azathioprine, methotrexate and 2-chlorodeoxyadenosine has also been used.

The final talk in the session was provided by Dr Rachel Hoyles (Oxford, UK) discussing the management and prognosis of connective tissue disease-related ILD, especially scleroderma. Severe disease is indicated by a baseline transfer factor of the lung for carbon monoxide (TLCO) less than 50\% predicted or forced vital capacity less than 55\%. A recent deterioration with a greater than $15 \%$ drop in the TLCO from baseline or most recent recording should prompt consideration of treatment. The evidence suggests the use of monthly intravenous cyclophosphamide for 6 months and then oral azathioprine or mycophenolate mofeteil. The use of high-dose steroids is to be avoided in case of precipitation of renal crises. More recently some small studies have suggested the efficacy of rituximab in rapidly progressive disease. $^{39}$

\section{THE BTS/BLF/BALR EARLY CAREER INVESTIGATOR AWARDS}

As always, the standard of research at the Early Career Investigator presentation was formidable. Thompson et al delivered a clear presentation showing the importance of HIF-2a in the resolution of neutrophilia from the site of inflammation. Using zebrafish as an animal model in which there were gain-of-function HIF-2a mutations, delayed resolution of inflammation following tail injury was observed. In addition, in a myeloid-specific HIF-2a knockout murine model of acute lung injury, there was increased clearance of neutrophils and less lung injury observed. These findings suggest that HIF-2a is important in the process of resolution of inflammation. ${ }^{40}$

Sage et al received the BALR prize for their presentation looking at MSCs as a mechanism for transporting a targeted therapy for inducing apoptosis in malignant cells. Sage elegantly described how the MSCs were transfected successfully with a lentivirus expressing TRAIL and that coculture of these cells with human malignant mesothelioma cell lines resulted in apoptosis of the malignant cells. In a mesothelioma in vivo animal model, evidence was presented that when these transfected MSCs were injected into the pleural cavity the cells homed to the tumour and there was significant reduction in the tumour growth. ${ }^{6}$

Puthucheary et al presented important results outlining the mechanisms underpinning muscle breakdown in the critically ill patient and was awarded the BTS prize. Using a variety of clinical and biochemical techniques, including muscle biopsy, they demonstrated that muscle loss occurs early in the illness and is more severe in multi-organ failure compared with single-organ failure. The cellular processes involved included decreased protein synthesis, increased myonecrosis and increased protein catabolism. $^{41}$

The presentation by Soon et al gave substantial evidence for the link between inflammation and pulmonary hypertension.
In particular, the role of BMPR2 gene mutations as proinflammatory were explored in murine and human models. Low-dose lipopolysaccharide given to BMPR2+/- mice led to the development of pulmonary hypertension mirrored with an increase in lung and serum IL-6. Increased oxidative stress with increased superoxide generation and reduced SOD3 levels was presented as a mechanism to explain this. Indeed coadministration of tempol, a SOD3 mimietic, helped to prevent the development of pulmonary hypertension in the BMPR 2 model. ${ }^{42}$

Jackson et al showed that during rhinovirus-induced asthma exacerbations the levels of IL-33 increase compared with nonasthmatic controls. This potentially then contributes to the activation of downstream $\mathrm{T}$ helper 2 inflammation and levels of IL-33 correlated with chest symptoms. This group also introduced the technique of 'bronchosorption', during which filter paper introduced at bronchoscopy can be used to sample the airway lining of distal airways and can avoid the dilution effect seen when trying to analyse for cytokines following bronchoalveolar lavage. ${ }^{43}$

Harrison et al were awarded the British Lung Foundation (BLF) prize for their presentation of a prospective cohort study examining the prognostic significance of thrombocytosis in acute exacerbation of chronic obstructive pulmonary disease (COPD). As many as $11 \%$ of the cohort had an elevated platelet count and this seemed to correlate with an increased mortality in hospital and in the year following discharge. The mechanism leading to this correlation is unclear. There was a suggestion that antiplatelet therapy conferred some benefit, although this needs to be studied in more detail. ${ }^{44}$

\section{LIFESTYLE AND THE RESPIRATORY PATIENT}

Edward Stanley, the earl of Derby, wrote in 1873: 'Those who think they have not time for bodily exercise will sooner or later have to find time for illness.' But questions of whether patients with respiratory disease should exercise, how to measure such activity or inactivity and how much should they exercise was addressed in a dedicated symposium. Most data on physical activity in respiratory disease is in the context of COPD. Dr Nicholas Hopkinson (London) discussed the importance of physical activity in COPD, with physical inactivity being associated with disease progression and adverse outcomes. Physical inactivity and muscle loss are evident even in early COPD ${ }^{45}$ and inactivity is associated with shorter time to first hospital admission with COPD, ${ }^{45}$ elevated inflammatory indices ${ }^{46}$ and poorer survival. ${ }^{47}$ In addition, evidence clearly shows interventions to enhance physical activity (pulmonary rehabilitation), reduce hospital admissions and improve quality of life in COPD. Dr Charlotte Bolton (Nottingham) updated the BTS regarding the draft version of the new BTS pulmonary rehabilitation guidelines. This updates the original 2001 guidelines ${ }^{49}$ and focuses on adults with COPD. The guidelines incorporate advances in our knowledge in this area over the past decade, including a review of the evidence regarding repeated rehabilitation, post-exacerbation rehabilitation and maintenance programmes. The draft guidelines are now available for public consultation (http://www.brit-thoracic.org.uk/guidelines/ pulmonary-rehabilitation-guidelines.aspx), with the aim of publication in 2013. Finally, Dr Thierry Trooster continued this theme via his discussion of the PROactive project (Physical Activity as a Crucial Patient Reported Outcome in COPD) which aims to develop and validate patient-reported outcome tools that can be used to investigate the aspects of physical activity which are important to the patient. To date, this multicentre European collaboration has compared the validity of activity monitors for use in COPD. ${ }^{50}$ 
A separate, well attended symposium explored the current evidence and controversies in approaching lifestyle dilemmas arising in clinical practice in patients with respiratory disease. Professor Adrain Williams (London) discussed the issues surrounding the sleepy driver, highlighting the prevalence of sleepiness in drivers, with $29-55 \%$ reporting drowsiness while driving and $11-31 \%$ having fallen asleep at the wheel. Evidence clearly links sleepiness and motor vehicle accidents (MVAs), with approximately $20 \%$ of MVAs being sleep related. He provided evidence of the increased risk in the context of obstructive sleep apnoea (OSA), with a 2.5 relative risk of an MVA in OSA, ${ }^{51}$ and that continuous positive airway pressure significantly reduces this risk. ${ }^{52}$ However, he highlighted that there is no reliable objective test that is predictive of increased driving risk and that prevention should be through education of drivers, likening the sleepy driver to be equivalent to the alcohol-intoxicated driver.

Recreational scuba diving is one of the fastest growing water sports worldwide and medical practitioners are increasingly likely to face requests concerning fitness to dive from respiratory patients. Dr Neil Martin (Leicester) was well qualified to inform the BTS on this issue as diving medical examiner with the Royal Navy, discussing the physiology of diving and the paucity of evidence examining diving and respiratory disease. There are several factors that may cause bronchospasm in the diving environment and up to $50 \%$ of military divers have positive direct bronchial challenge tests. ${ }^{53}$ Current guidelines outline an approach to assess patients with asthma, ${ }^{54} 55$ but diving in the context of, potentially undiagnosed, COPD may be an increasingly realistic issue. However, Dr Martin highlighted that there is very little evidence from accident statistics that obstructive airways disease is associated with increased risk.

Each year over 2 billion people travel by air worldwide and over 200 million travel alone in the UK. Dr Mark Elliot (Leeds) gave an entertaining and practical discussion of air travel in people who use ventilators. He highlighted limitations of using the hypoxic challenge test (HCT) alone in assessing patients with restrictive lung disease $e^{5-58}$ and outlined his approach combining HCT, previous flight experience, stability of disease and timing/duration of the flight. From the patient perspective, it was clear that flying is possible for those who use ventilators but requires careful forward planning of the flight and the logistics of travelling. Professor David Gradwell (London) described the physiological effects of flying on patients with respiratory conditions, and again, highlighted that the HCT is not infallible. Describing the frequency of adverse medical events in the air (with medical incidents as frequent as 1:750 flights) and the effects of decompression illness, Professor Gradwell concluded the session by putting us all in trepidation of our flights home!

\section{CONCLUSION}

The annual BTS Winter Meeting has an outstanding reputation for bringing together healthcare providers, researchers and scientists and in disseminating cutting edge respiratory research. The BTS 2012 Meeting continued this high standard and has taken us through a wide spectrum of respiratory research, from the cellular and subcellular level to the underwater and highaltitude environments.

Contributors Review research and writing: $\mathrm{HKB}, \mathrm{ACC}$ and $\mathrm{AF}$; concept: AF. Competing interests None.

Provenance and peer review Not commissioned; internally peer reviewed.

\section{REFERENCES}

1 Melchor R, Biddiscombe MF, Mak VH, et al. Lung deposition patterns of directly labelled salbutamol in normal subjects and in patients with reversible airflow obstruction. Thorax1993;48:506-11.

2 Mak V, Marlow H, Restrick L, et al. Are healthcare professionals aware of costs of respiratory inhalers? Thorax 2012;67(Suppl2):A170.

3 Usmani OS, Biddiscombe MF, Barnes PJ. Regional lung deposition and bronchodilator response as a function of $\beta 2$-agonist particle size. Am J Respir Crit Care Med 2005:172:1497-504.

4 Usmani OS. Treating the small airways. Respiration 2012;84:441-53.

5 Beverly A, Tetley TD, Thorley AJ. Evaluation and treatment of cystic fibrosis: S125 Pro-fibrotic effects of multi-walled carbon nanotube exposure on primary human alveolar type II epithelial cells and fibroblasts. Thorax 2012;67(Suppl 2):A60. doi:10.1136/thoraxjnl-2012-202678.130

6 Sage EK, McNulty K, Kolluri K, et al. T2 MSCTRAIL as a novel cellular therapy for malignant mesothelioma. Thorax 2012;67(Suppl 2):A1. doi:10.1136/ thoraxjnl-2012-202678.002

7 Kwak EL, Bang Y-J, Camidge DR, et al. Anaplastic lymphoma kinase inhibition in non-small-cell lung cancer. N Engl J Med 2010;363:1693-703.

8 Shaw AT, Yeap BY, Solomon BJ, et al. Effect of crizotinib on overall survival in patients with advanced non-small-cell lung cancer harbouring ALK gene rearrangement: a retrospective analysis. Lancet Oncol Elsevier 2011; 12:1004-12.

9 O'Neill S, Sweeney J, Neill CO, et al. Severe asthma and exacerbations: S64 The cost of refractory asthma in the UK - a preliminary analysis. Thorax 2012;67(Suppl 2):A32. doi:10.1136/thoraxjnl-2012-202678.070

10 Sweeney J, Brightling CE, Menzies-Gow A, et al. Clinical management and outcome of refractory asthma in the UK from the British Thoracic Society Difficult Asthma Registry. Thorax 2012;67:754-6.

11 Jia G, Erickson RW, Choy DF, et al. Periostin is a systemic biomarker of eosinophilic airway inflammation in asthmatic patients. J Allergy Clin Immunol 2012;130:647-654.e10.

12 Corren J, Lemanske RF Jr., Hanania NA, et al. Lebrikizumab treatment in adults with asthma. N Engl J Med 2011;365:1088-98.

13 Bicknell S, Chaudhuri R, Shepherd M, et al. Treating asthma: P5 Introducing bronchial thermoplasty treatment into a severe asthma clinical service. Thorax 2012;67(Suppl 2):A65-6. doi:10.1136/thoraxjnl-2012-202678.146

14 Bel EH, Sousa A, Fleming $L$, et al. Diagnosis and definition of severe refractory asthma: an international consensus statement from the Innovative Medicine Initiative (IMI). Thorax 2011;66:910-17.

15 Maskell NA, Davies CWH, Nunn AJ, et al. U.K. controlled trial of intrapleural streptokinase for pleural infection. N Engl J Med 2005;352:865-74.

16 Light RW, Nguyen T, Mulligan ME, et al. The in vitro efficacy of varidase versus streptokinase or urokinase for liquefying thick purulent exudative material from loculated empyema. Lung 2000;178:13-18.

17 Simpson G, Roomes D, Heron M. Effects of streptokinase and deoxyribonuclease on viscosity of human surgical and empyema pus. Chest 2000;117:1728-33.

18 Rahman NM, Maskell NA, West A, et al. Intrapleural use of tissue plasminogen activator and DNase in pleural infection. N Engl J Med 2011;365:518-26.

19 Grijalva CG, Zhu Y, Nuorti JP, et al. Emergence of parapneumonic empyema in the USA. Thorax 2011;66:663-8.

20 Lisboa T, Waterer GW, Lee YCG. Pleural infection: changing bacteriology and its implications. Respirology 2011;16:598-603.

21 Tobin CL, Lee YCG. Pleural infection. Curr Opin Pulm Med 2012;18:321-5.

22 Wilkosz S, Edwards LA, Bielsa S, et al. Characterization of a new mouse model of empyema and the mechanisms of pleural invasion by streptococcus pneumoniae. Am J Respir Cell Mol Biol 2012;46:180-7

23 Menzies SM, Rahman NM, Wrightson JM, et al. Blood culture bottle culture of pleural fluid in pleural infection. Thorax 2011;66:658-62.

24 Maskell NA, Batt S, Hedley EL, et al. The bacteriology of pleural infection by genetic and standard methods and its mortality significance. Am J Respir Crit Care Med 2006;174:817-23.

25 Thomas MF, Sheppard CL, Guiver M, et al. Emergence of pneumococcal 19A empyema in UK children. Arch Dis Child 2012;97:1070-2.

26 Pelton $\mathrm{SI}$, Huot $\mathrm{H}$, Finkelstein JA, et al. Emergence of $19 \mathrm{~A}$ as virulent and multidrug resistant Pneumococcus in Massachusetts following universal immunization of infants with pneumococcal conjugate vaccine. Pediatr Infect Dis $J$ 2007;26:468-72

27 Corcoran JP, Lockey JW, Zheng SL, et al. S13 The bacteriology and epidemiology of pleural infection-a review of the literature and its relevance to clinical practise. Thorax 2012;67(Suppl 2):A9. doi:10.1136/thoraxjnl-2012-202678.019.

28 Pitman N, McCartney R, O'Dowd C, et al. S12 Experience with intrapleural tissue plasminogen activator and DNase in the treatment of pleural infection. Thorax 2012:67(Suppl 2):A8-9.

29 Hooper CE, Edey AJ, Wallis AJ, et al. S17 Pleural Irrigation Trial (PIT): standard care versus pleural irrigation, a randomised controlled trial in patients with pleural infection. Thorax 2012;67(Suppl 2):A11. doi:10.1136/thoraxjnl-2012-202678.023 
30 Wilkinson A, Lok SS, Thomas EC. S14 The best way to secure a 12-French intercostal chest drain to the chest wall. Thorax 2012;67(Suppl 2):A9. doi:10.1136/ thoraxjnl-2012-202678.020

31 Buckley B, Haris M, Miller B, et al. Pleural disease: S15 is contrast-enhanced Computed Tomography (CT) scoring a useful tool in predicting pleural malignancy on thoracoscopy? Thorax 2012;67(Suppl 2):A10. doi:10.1136/ thoraxjnl-2012-202678.021

32 Navaratnam V, Fogarty A, McKeever T, et al. Clinical studies in interstitial lung disease: S97 Is an increased tendency to clot a risk factor for developing idiopathic pulmonary fibrosis? Thorax 2012;67(Suppl 2):A48. doi:10.1136/thoraxjn-2012-202678.102

33 Noth I, Anstrom KJ, Calvert SB, et al. A placebo-controlled randomized trial of warfarin in idiopathic pulmonary fibrosis. Am J Respir Crit Care Med 2012;186:88-95.

34 Mann J, Chu DCK, Maxwell A, et al. MeCP2 controls an epigenetic pathway that promotes myofibroblast transdifferentiation and fibrosis. Gastroenterology 2010;138:705-14.e4

35 Parfrey H, Leonard C, Gibbons MA, et al. Clinical studies in interstitial lung disease: S98 early clinical experience with pirfenidone for idiopathic pulmonary fibrosis (IPF) in the UK: interim results from a UK cohort. Thorax 2012;67(Suppl 2):A48-9. doi:10.1136/thoraxinl-2012-202678.103

36 McCormack FX, Inoue Y, Moss J, et al. Efficacy and safety of sirolimus in lymphangioleiomyomatosis. N Engl J Med 2011;364:1595-606.

37 Yeoh ZW, Navaratnam V, Hubbard RB, et al. Clinical studies in interstitial lung disease: S100 natural history of renal angiomyolipoma in sporadic and tuberous sclerosis complex associated lymphangioleiomyomatosis: implications for clinical care. Thorax 2012;67(Suppl 2):A49. doi:10.1136/thoraxjnl-2012-202678.105

38 Tazawa R, Trapnell BC, Inoue Y, et al. Inhaled granulocyte/macrophage-colony stimulating factor as therapy for pulmonary alveolar proteinosis. Am J Respir Crit Care Med 2010;181:1345-54.

39 Daoussis D, Liossis S-NC, Tsamandas AC, et al. Experience with rituximab in scleroderma: results from a 1-year, proof-of-principle study. Rheumatology (Oxford) 2010;49:271-80

40 Thompson A, Elks PM, Marriott HM, et al. T1 hypoxia-inducible factor $2 \alpha$ regulates neutrophilic inflammation in humans, mice and zebrafish. Thorax 2012;67(Suppl 2): A1. doi:10.1136/thoraxinl-2012-202678.001

41 Puthucheary ZA, Rawal J, Mcphail M, et al. T3 acute muscle loss in the critically III: from bedside to bench. Thorax 2012;67(Suppl 2):A1-2. doi:10.1136/ thoraxjnl-2012-202678.003

42 Soon $\mathrm{E}$, Crosby $\mathrm{A}$, Southwood M, et al. T4 mutations in BMPR-II promote inflammation via altered superoxide signalling: insights into the mechanisms underlying pulmonary arterial hypertension. Thorax 2012;67(Suppl 2):A2-3. doi:10.1136/thoraxjnl-2012-202678.004

43 Jackson DJ, Trujillo-Torralbo M, Footitt J, et al. T5 sampling airway mucosal lining fluid identifies roles for IL-33 and multiple inflammatory pathways in virus-induced asthma exacerbations. Thorax 2012:67(Suppl 2):A3. doi:10.1136/ thoraxjnl-2012-202678.005

44 Harrison MT, Short P, Williamson P, et al. T6 thrombocytosis is associated with increased short and long term mortality after exacerbation of chronic obstructive pulmonary disease. Thorax 2012;67(Suppl 2):A3. doi:10.1136/ thoraxjnl-2012-202678.006

45 Shrikrishna D, Patel M, Tanner RJ, et al. Quadriceps wasting and physical inactivity in patients with COPD. Eur Respir J 2012;40:1115-22.

46 Garcia-Aymerich J. Physical activity and clinical and functional status in COPD. Chest 2009;136:62.

47 Garcia-Aymerich J, Lange $\mathrm{P}$, Benet $\mathrm{M}$, et al. Regular physical activity reduces hospital admission and mortality in chronic obstructive pulmonary disease: a population based cohort study. Thorax 2006;61:772-8.

48 Waschki B. Physical activity is the strongest predictor of all-cause mortality in patients with COPDPhysical activity and all-cause mortality in COPDA prospective Cohort Study. Chest Am Coll Chest Phys 2011;140:331-42.

49 British Thoracic Society Standards of Care Subcommittee on Pulmonary Rehabilitation. Pulmonary rehabilitation. Thorax 2001;56:827-34.

50 Van Remoortel H, Raste $Y$, Louvaris Z, et al. Validity of six activity monitors in chronic obstructive pulmonary disease: a comparison with indirect calorimetry. PLOS ONE 2012;7:e39198.

51 Stephen Tregear JRKSBP. Obstructive sleep apnea and risk of motor vehicle crash: systematic review and meta-analysis. J Clin Sleep Med 2009;5:573.

52 Antonopoulos CN, Sergentanis TN, Daskalopoulou SS, et al. Nasal continuous positive airway pressure (nCPAP) treatment for obstructive sleep apnea, road traffic accidents and driving simulator performance: a meta-analysis. Sleep Med Rev 2011;15:301-10.

53 Tetzlaff K, Neubauer B, Reuter M, et al. Atopy, airway reactivity and compressed air diving in males. Respiration 1998;65:270-4.

54 British Thoracic Society Scottish Intercollegiate Guidelines Network. British guideline on the management of asthma. Thorax 2008;63:iv1-121.

55 Turner S, Paton J, Higgins B, et al. British guidelines on the management of asthma: what's new for 2011? Thorax 2011;66:1104-5.

56 Shrikrishna D, Coker RK, on behalf of the Air Travel Working Party of the British Thoracic Society Standards of Care Committee. Managing passengers with stable respiratory disease planning air travel: British Thoracic Society recommendations. Thorax 2011;66:831-3.

57 Ahmedzai S, Balfour-Lynn IM, Bewick T, et al. Managing passengers with stable respiratory disease planning air travel: British Thoracic Society recommendations. Thorax 66(Suppl 1):i1.

58 Mestry N, Thirumaran M, Tuggey JM, et al. Hypoxic challenge flight assessments in patients with severe chest wall deformity or neuromuscular disease at risk for nocturnal hypoventilation. Thorax 2009;64:532-4. 